\title{
Epigenetic regulation of human embryonic stem cells
}

\section{Qidong Hu* and Michael G. Rosenfeld*}

School/Department of Medicine, Howard Hughes Medical Institute, University of California, San Diego, CA, USA

\section{Edited by:}

Halyna R. Shcherbata, Max Planck

Society, Germany

\section{Reviewed by:}

Ian C. Weaver, Centre for Addiction and Mental Health, Canada

Halyna R. Shcherbata, Max Planck

Society, Germany

\section{${ }^{*}$ Correspondence:}

Qidong Hu and Michael G. Rosenfeld, School/Department of Medicine,

Howard Hughes Medical Institute, University of California, San Diego, CA, USA.

e-mail: qihu@ucsd.edu;

mrosenfeld@ucsd.edu
Recently, there has been tremendous progress in characterizing the transcriptional network regulating human embryonic stem cells (hESCs; MacArthur etal., 2009; Loh et al., 2011), including those signaling events mediated by Oct4, Nanog, and Sox2. There is growing interest in the epigenetic machinery involved in hESC self-renewal and differentiation. In general, epigenetic regulation includes chromatin reorganization, DNA modification, and histone modification, which are not directly related to alterations in DNA sequences. Various protein complexes, including Polycomb, trithorax, nucleosome remodeling deacetylase, $\mathrm{SWI} / \mathrm{SNF}$, and Oct4, have been shown to play critical roles in epigenetic control of hESC physiology. Hence, we will formally review recent advances in unraveling the multifaceted role of epigenetic regulation in hESC self-renewal and induced differentiation, particularly with respect to chromatin remodeling and DNA methylation events. Elucidating the molecular mechanisms underlying the maintenance/differentiation of hESCs and reprogramming of somatic cells will greatly strengthen our capacity to generate various types of cells to treat human diseases.

Keywords: embryonic stem cell, epigenetic regulation, DNA methylation, chromatin remodeling, DNA repeat

\section{CHROMATIN REMODELING IN hESCs}

Human embryonic stem cells (hESCs) feature the capability to self-renew and to differentiate into all types of cells in humans. Hence, they are therapeutically invaluable to treat major human diseases, such as neurodegeneration, diabetes, and cardiovascular diseases. Recently, various approaches have been developed to reprogram human somatic cells into induced pluripotent stem cells (iPS cells; Yamanaka, 2009), providing a more abundant and ethical-feasible source of progenitor cells that possess the capacity of generating all types of human cells. However, the low efficiency of reprogramming terminally differentiated cells into iPS cells has remained a major obstacle that prevents the wide application of this technology in practical use. Hence, to achieve a more comprehensive understanding of hESC biology and to promote application of the stem cell strategy in treating devastating diseases, scientists have begun to explore new territories in the context of hESC maintenance/differentiation, including the role of chromatin remodeling.

It is well established that chromatin is composed of the DNA component, the histone components being wrapped and other related proteins. The dynamic assembly or disassembly of DNA-histone structure is closely associated with many important biological processes, such as DNA replication, DNA repair, and transcription. Previous studies have shown that in hESCs, the chromatin is in a less compact state globally than in terminally differentiated cells (Meshorer et al., 2006). This loose compaction of chromatin facilitates more dynamic and flexible reorganization during differentiation.

Many complexes, collectively referred to as chromatin remodelers that are mostly ATP-dependent, have been identified to contribute to this less compact state. Chromatin remodelers mediate the interaction between the DNA helix and histones, hence regulating the accessibility of the DNA to transcription factors and other machinery (Ho and Crabtree, 2010). Some wellcharacterized remodeling complexes include SWI/SNF, NuRD, Tip60/p400, and chromodomain helicase DNA binding protein (CHD), which will be detailed in the following.

The SWI/SNF complex contains 9-12 subunits, among which BAFs (including BAF47 and BAF155/170) play critical regulatory roles (Phelan etal., 1999) while Brg1 and Brm possess the ATPase activity (de la Serna et al., 2006). Both Brgl and Brm contain bromodomains, hence they exhibit a preference for acetylated histones. These proteins are ubiquitously utilized in mammalian development, both in the maintenance of stem cell state and in differentiation. In particular, Brg1 has been shown to interact with the key regulators of pluripotency, Oct4, Sox2, and NANOG, and exhibits a highly correlated genome-wide binding patterns with these proteins (Liang et al., 2008; Ho et al., 2009), suggesting a cooperative role of SWI/SNF complexes in keeping the cells in the undifferentiated state. In addition, Brg1 binds to many development-related genes exhibiting bivalent histone marks that represent a "poised" status of activation. The bivalent histone mark includes the $\mathrm{H} 3 \mathrm{~K} 4 \mathrm{me} 3$ activation and the $\mathrm{H} 3 \mathrm{~K} 27 \mathrm{me} 3$ repressive modifications, which are particularly confined to embryonic stem cells (Bernstein et al., 2006). Hence Brg1 coverage of these regions may ensure an efficient and reversible repression of differentiation-related genes. Once differentiation is irreversibly initiated, BAF155 increases the H3K27me3 modification at the NANOG promoter and consequently causes condensation of the local chromatin, hence repressing the expression of NANOG (Schaniel et al., 2009). If BAF155 is depleted, the expression level of NANOG will remain high so that differentiation of hESCs will be significantly impaired. Additionally, genome-wide location analysis has found that BAF155 is also involved in depositing on chromatin the $\mathrm{H} 3 \mathrm{~K} 9$ me 3 mark, a modification that contributes to heterochromatin formation (Schaniel et al., 2009). Hence, even 
though co-existed in one SWI/SNF complex, Brg1 and BAF155 exert somewhat opposing effects on stem cell maintenance and differentiation.

The Mi-2/nucleosome remodeling deacetylase (NuRD) complex possesses both the APT-dependent chromatin remodeling activity and the histone deacetylase activity (Denslow and Wade, 2007). The key components include chromodomain helicase CHD3/4, deacetylase HDAC1/2, methy-CpG-binding proteins $\mathrm{Mbd} 3$ and Mta1. They are involved in regulating pluripotency and differentiation of ESCs via histone deacetylation. The deficiency of Mbd3 leads to hyperacetylation and loss of ES pluripotency (Zhu et al., 2009). For a similar complex that lacks Mbd3, NODE, studies have found that it interacts with NANOG and Oct4, and co-binds to the NANOG/Oct4 target genes (Liang et al., 2008). Depletion of Mtal de-represses genes related to endoderm differentiation, such as GATA6 and FoxA2 (Liang et al., 2008), indicating that an intact NODE complex is required for suppression of premature cell lineage commitment.

The Tip60/p400 complex also contains both an ATPase activity and an acetyltransferase activity. In particular, the ATPase activity is conferred by p400. Both activities are required for early embryonic development (Fazzio et al., 2008; Hu et al., 2009). Depletion of Tip60/p400 leads to de-repression of genes, many of which are important developmental regulators. Down-regulation of this complex also impairs self-renewal of progenitor cells. Consistently, p400 binding profile coincides with those of H3K4me3 and bivalent marks, a unique epigenetic feature in ESCs (Fazzio et al., 2008), implying that the Tip60/p400 complex is involved in maintaining the pluripotent state of the cell.

A fourth major remodeling family is the CHDs. They contain two chromodomains, hence exhibiting high affinity for methylated histones, especially $\mathrm{H} 3 \mathrm{~K} 4 \mathrm{me} 2 / 3$ (Flanagan et al., 2005; Marfella and Imbalzano, 2007; Sims et al., 2007). One member of particular significance is CHD1, which has been proved to be required for maintaining a loose/open chromatin conformation in ESCs (Gaspar-Maia et al., 2009). Depletion of CHD1 leads to heterochromatin formation characterized by high enrichment of $\mathrm{H} 3 \mathrm{~K} 9 \mathrm{me} 3$ and HP1 $\gamma$, down-regulation of Oct4, and initiation of neural development (Gaspar-Maia et al., 2009). Another major member, CHD7, has been found critical for generation of migration-competent neural crest-like cell from hESCs (Bajpai et al., 2010). In humans, mutation of CHD7 can also cause a genetic disorder, CHARGE, which is characterized by severe defects in many cell types at birth (Vissers et al., 2004), implying that CHD7 is indeed involved in embryonic development. It is interesting to note that $\mathrm{CHD} 7$ harbors the BRK domain, which is known to bind to CTCF (Allen et al., 2007), the protein playing as insulators, hence raising the possibility that $\mathrm{CHD} 7$ may help create a chromatin landscape with active (self-renewal and pluripotency-related genes) and quiescent (cell lineage-specific genes) segments.

\section{DNA METHYLATION REGULATION IN hESC MAINTENANCE AND DIFFERENTIATION}

DNA methylation mainly occurs at the 5-C position of the CG dinucleotide in mammalian cells, the occurrence of which is inversely correlated with the GC content and CpG density (Bird,
2002; Illingworth and Bird, 2009). It represents a major epigenetic regulation for many biological processes, such as gene transcription, imprinting and transposon activity in embryonic stem cells, germ cells, somatic cells, and tumor cells (Aranyi and Paldi, 2006; Farthing et al., 2008; Xie et al., 2009).

DNA methylation is accomplished by three independent DNA methyltransferases, DNMT1, DNMT3A, and DNMT3B (Kato et al., 2007). In particular, DNMT3A and 3B are responsible for de novo methylation events at cysteine while DNMT1 is involved in the maintenance of the methylated status (Okano et al., 1999; Chen et al., 2003). Depletion of DNMT members leads to embryonic lethality in the mouse model (Li et al., 1992; Okano et al., 1998), and in in vitro culture, even though DNMT-knockout cells can still self-renew, they lose the potentiality to differentiate (Thomson et al., 1998; Okano et al., 1999), suggesting that DNMT plays a more prominent role in pluripotency. DNA methylation plays a critical epigenetic role in terms of gene expression (Loh et al., 2011) and has different patterns in hESCs and differentiated cells. In hESCs, the whole genome is relatively hypomethylated, reflecting a more open and hyperdynamic nature of chromatin, while in the latter, there is more DNA methylation genomewide suggesting a highly organized and a less active chromatin conformation (Robertson, 2001; Bibikova et al., 2006; Gan et al., 2007). Previous research has revealed an interesting inverse correlation between $\mathrm{CpG}$ methylation and $\mathrm{CpG}$ dinucleotide density, whereby densely packed $\mathrm{CpG}$ islands appear to be protected from methylation (Kafri et al., 1992). Generally, CpG islands near promoters of actively expressed genes are demethylated and methylation at these sites is typically associated with gene repression during development (Bird, 2002). However, methylation within the gene is usually associated with active transcription of the gene, indicating that DNA methylation at different regions of genes can play opposite roles (Laurent et al., 2010). In hESCs, the CpG islands in the promoters of housekeeping genes and key stem cell signature genes, such as Oct4, NANOG, TDGF1, and Sox2, remain demethylated, hence permitting their active expression to maintain the stem cell state (Weber etal., 2007; Meissner et al., 2008). This notion is strengthened by studies using the DNA methyltransferase inhibitors, 5-azacytidine and RG108, in which cases the efficiency of reprogramming mouse fibroblasts to iPS cells were greatly enhanced (Mikkelsen et al., 2008; Shi et al., 2008).

In a recent study, a substantial amount of non-CpG methylation was also uncovered in hESCs (Lister et al., 2009). However, these non-conventional methylation simply disappeared upon induced differentiation, indicating that ESCs uniquely employ an additional methylation strategy to epigenetically regulate gene expression. By pairwise comparison of hESC and fetal fibroblasts, the same group also observed a differentiation-related decrease in $\mathrm{CpG}$ methylation as well as a positive correlation between $\mathrm{CpG}$ methylation level in the gene body and gene expression in differentiated cells.

In addition, DNA methylation occurred in repetitive sequences has started to draw increasing attention from the field, partially due to the fact that in human cells, gene-coding regions only takes up $\sim 2 \%$ of the whole genome (Lander et al., 2001), while $\sim 47 \%$ of the DNA content can be classified into different types of repetitive 
elements - long-interspersed element (LINE), short-interspersed element (SINE), SVA, LTR, satellite repeats, etc. A typical LINE repeat occupies a $\sim 6 \mathrm{~kb}$ DNA sequence. In the human genome, LINE repeats are mainly located outside coding sequences of genes, such as promoters, introns, and untranslated regions. In hESCs, they are constitutively transcribed into RNAs. Two open reading frames, ORF1 and ORF2 (Scott et al., 1987), cannot only be transcribed into RNAs, but also produce retrotranscriptase that is essential for transposition of other retrotransposons, such as Alu repeats. Additionally, ORF1 is transcribed and translated into a protein with RNA binding and chaperon activity (Hohjoh and Singer, 1997; Martin and Bushman, 2001). Alu repeats are evolved from the 7SL RNA, and are typically $300 \mathrm{bp}$ in length. There are about one million copies of Alu repeats which account for $\sim 11 \%$ DNA contents of the human genome (Rubin et al., 1994). They are scattered within both coding and non-coding regions. In general, these repetitive DNA elements had once been referred to as "junk DNA" since no definitive functions had been uncovered for them. However, recent work has suggested that they represent an overlooked "treasure chest" with huge regulatory potentiality to exert tremendous influence on cells, including the integrity of human genome, embryogenesis, and tumorigenesis.

Significantly, over half of CpG methylation events in the human genome is distributed among various types of DNA repeats (Cordaux and Batzer, 2009), but the methylation density varies greatly among different classes of DNA repeats. In particular, about onethird of the $\mathrm{CpG}$ islands are embedded in Alu repeats which exhibit CpG density-correlated methylation, whereas CpG islands embedded in LINE and LTR elements are generally hypermethylated regardless of the GC content (Meissner et al., 2008).

Hypermethylation contributes to silencing of these repeats and formation of heterochromatin. Since LINE, Alu and SVA repetitive elements are frequently located in proximity to genes with protein coding capacities (Cordaux and Batzer, 2009), these inhibitory effects will most likely spread to the adjacent genes, thus epigenetically regulating gene expression in hESCs. Additionally, in terminally differentiated human cells, repetitive elements are generally highly methylated and the quiescence of these repeats is required for the integrity and stability of the cell genome (Donnelly et al., 1999; Kato et al., 2007).

Recent studies have found that many repetitive elements are hypermethylated in human pluripotent cells as compared to differentiated cells (Meissner et al., 2008; Su et al., 2012), suggesting an active role of these elements in maintaining the stem cell state. The genome-wide analysis has shown that cells experience dramatic changes in DNA methylation pattern during cell fate commitment (Meissner et al., 2008; Lister et al., 2009; Nagae et al., 2011). In particular, LINE, LTR, and satellite elements show a more significant differentiation-induced decrease in CpG methylation than SINE, RNA repeats, and SVA repeats (Su et al., 2012). This differential demethylation pattern can be partly explained by the relative lower $\mathrm{CpG}$ density along the DNA sequences of LINE and LTR (Su et al., 2012). Their study has further found that the demethylated repetitive elements are mainly distributed in intergenic regulatory regions and introns, but not in coding exons (Meissner et al., 2008; Su et al., 2012), suggesting a potential epigenetic regulation of the adjacent coding region.
Indeed, genomic profiling of the demethylated repetitive elements has shown that most of the intron-embedded repeats are methylated in hESCs but demethylated in differentiated cells. The genes containing demethylated repetitive elements in their introns generally exhibit a significantly lower level of expression, suggesting a synergized regulation of methylation in gene-embedded repeats and gene transcription (Ball et al., 2009; Su et al., 2012). Interestingly, in many human cancers, $\mathrm{CpG}$ hypomethylation has been observed in repetitive DNA elements, LINE, SINE, LTR, and satellite repeats (Rauch et al., 2008; Bollati et al., 2009; Choi et al., 2009; Igarashi et al., 2010; Xie et al., 2010), reflecting that tumorigenesis potentially shares some common epigenetic regulation pathways with hESC maintenance and somatic cell reprogramming.

\section{CROSSTALK BETWEEN HISTONE MODIFICATION AND DNA METHYLATION}

Histone modifications represent another major field of research on epigenetic regulation of gene expression, and there are many excellent reviews recently published (Kato et al., 2011; Loh et al., 2011). Hence, in the paper, we instead focus on the crosstalk between DNA methylation and histone modification.

Studies have identified an inverse relationship between $\mathrm{H} 3 \mathrm{~K} 4$ methylation and DNA methylation (Hashimshony et al., 2003; Meissner etal., 2008). One study observed that methylation at $\mathrm{CpG}$-dense regions will inhibit the local Trithorex activity that confers H3K4 methylation modification (Bird, 2002). Since $\mathrm{H} 3 \mathrm{~K} 4 \mathrm{me} 3$ is usually associated with gene activation and present at $\mathrm{CpG}$ islands largely enriched in promoter regions, these mutually exclusive effects may be part of the coordinative mechanism underlying gene activation and silencing.

At the protein level, an interaction between methylcytosine binding proteins, $\mathrm{MeCP} 2 / \mathrm{Mbd} 2$, and $\mathrm{HDAC} / \mathrm{HMT}$ has been observed (Jones et al., 1998; Nan etal., 1998; Fuks et al., 2003). However, a causal relationship has not yet been established. There are also reports indicating that G9a and ESET, two methyltransferases for $\mathrm{H} 3 \mathrm{~K} 9$, can interact with DNMT3A/3B to facilitate local methylation of DNA (Feldman et al., 2006; Li et al., 2006). EZH2, an enzymatic component of the PRC2 complex involved in H3K27 methylation, can also interact with DNMTs to influence the DNA methylation status (Viré et al., 2006).

\section{CONCLUDING REMARKS}

Recent studies on epigenetic landscape of embryonic stem cells have gradually unraveled another layer of regulation on pluripotency maintenance and differentiation. However, our current knowledge of the spatiotemporal alterations in epigenetic modifications that accompany ESC maintenance and transition/differentiation into functional somatic cells has remained very limited, which prevents us from drawing a comprehensive image of epigenetic regulatory network in hESCs. The crosstalk between different epigenetic machinery, chromatin remodeling, histone modifications, and DNA methylation, certainly warrants future explorations to elucidate their respective roles in the epigenetic regulation. We are also facing the challenge of integrating the transcriptional regulation network and epigenetic network to fully understand hESC self-renewal and differentiation. Furthermore, 
another major component of the central theorem, RNA, is gradually coming into sight regarding the pluripotency maintenance and development. Recent studies on non-coding RNAs and small RNAs have suggested that some RNA species could regulate CpG

\section{REFERENCES}

Allen, M. D., Religa, T. L., Freund, S. M., and Bycroft, M. (2007). Solution structure of the BRK domains from CHD7. J. Mol. Biol. 371, 1135-1140.

Aranyi, T., and Paldi, A. (2006). The constant variation: DNA methylation changes during preimplantation development. FEBS Lett. 580, 65216526.

Bajpai, R., Chen, D. A., Rada-Iglesias, A., Zhang, J., Xiong, Y., Helms, J., et al. (2010). CHD7 cooperates with PBAF to control multipotent neural crest formation. Nature 463, 958-962.

Ball, M. P., Li, J. B., Gao, Y., Lee, J. H., LeProust, E. M., Park, I. H., et al. (2009). Targeted and genome-scale strategies reveal gene-body methylation signatures in human cells. Nat. Biotechnol. 27, 361-368.

Bernstein, B. E., Mikkelsen, T. S., Xie, X., Kamal, M., Huebert, D. J., Cuff, J., et al. (2006). A bivalent chromatin structure marks key developmental genes in embryonic stem cells. Cell $125,315-326$.

Bibikova, M., Chudin, E., Wu, B., Zhou, L., Garcia, E. W., Liu, Y., et al. (2006). Human embryonic stem cells have a unique epigenetic signature. Genome Res. 16, 1075-1783.

Bird, A. (2002). DNA methylation patterns and epigenetic memory. Genes Dev. 16, 6-21.

Bollati, V., Fabris, S., Pegoraro, V., Ronchetti, D., Mosca, L., Deliliers, G. L., et al. (2009). Differential repetitive DNA methylation in multiple myeloma molecular subgroups. Carcinogenesis 30, 1330-1335.

Cernilogar, F. M., Onorati, M. C., Kothe, G. O., Burroughs, A. M., Parsi, K. M., Breiling, A., et al. (2011). Chromatinassociated RNA interference components contribute to transcriptional regulation in Drosophila. Nature 480 391-395.

Chen, T., Ueda, Y., Dodge, J. E., Wang, Z., and Li, E. (2003). Establishment and maintenance of genomic methylation patterns in mouse embryonic stem cells by Dnmt3a and Dnmt3b. Mol. Cell. Biol. 23, 5594-5605.

Choi, S. H., Worswick, S., Byun, H. M., Shear, T., Soussa, J. C., Wolff, E. M., et al. (2009). Changes in DNA methylation of tandem DNA repeats are different from interspersed repeats in cancer. Int. J. Cancer 125, 723-729.

Cordaux, R., and Batzer, M. A. (2009). The impact of retrotransposons on human genome evolution. Nat. Rev. Genet. 10, 691-703.

de la Serna, I. L., Ohkawa, Y., and Imbalzano, A. N. (2006). Chromatin remodelling in mammalian differentiation: lessons from ATP-dependent remodellers. Nat. Rev. Genet. 7 461-473.

Denslow, S. A., and Wade, P. A. (2007) The human Mi-2/NuRD complex and gene regulation. Oncogene 26 5433-5438.

Donnelly, S. R., Hawkins, T. E., and Moss, S. E. (1999). A conserved nuclear element with a role in mammalian gene regulation. Hum. Mol Genet. 8, 1723-1728.

Farthing, C. R., Ficz, G., Ng, R. K. Chan, C. F., Andrews, S., Dean, W., et al. (2008). Global mapping of DNA methylation in mouse promoters reveals epigenetic reprogramming of pluripotency genes. PLoS Genet. 4, e1000116. doi: 10.1371/journal.pgen.1000116

Fazzio, T. G., Huff, J. T., and Panning, B. (2008). An RNAi screen of chromatin proteins identifies Tip60-p400 as a regulator of embryonic stem cell identity. Cell 134, 162-174.

Feldman, N., Gerson, A., Fang, J., Li, E. Zhang, Y., Shinkai, Y., et al. (2006). G9a-mediated irreversible epigenetic inactivation of Oct-3/4 during early embryogenesis. Nat. Cell Biol. 8, 188-194.

Flanagan, J. F., Mi, L. Z., Chruszcz, M. Cymborowski, M., Clines, K. L., Kim Y., et al. (2005). Double chromodomains cooperate to recognize the methylated histone H3 tail. Nature 438, 1181-1185.

Fuks, F., Hurd, P. J., Wolf, D., Nan, X., Bird, A. P., and Kouzarides, T. (2003). The methyl-CpG-binding protein MeCP2 links DNA methylation to histone methylation. J. Biol. Chem. 278, 4035-4040.

Gan, Q., Yoshida, T., McDonald, O G., and Owens, G. K. (2007). Concise review: epigenetic mechanisms contribute to pluripotency and cell lineage determination of embryonic stem cells. Stem Cells 25, 2-9.

Gaspar-Maia, A., Alajem, A., Polesso, F., Sridharan, R., Mason, M. J., Heidersbach, A., et al. (2009). Chd1 regulates open chromatin and pluripotency of embryonic stem cells. Nature 460 863-868.

Hashimshony, T., Zhang, J., Keshet, I. Bustin, M., and Ceder, H. (2003). The

methylation at promoter regions and gene expression by interacting with DNMT (Cernilogar et al., 2011; Rajasethupathy et al., 2012), adding another twist to the already exciting research field of epigenetic regulation in hESCs.

role of DNA methylation in setting up chromatin structure during development. Nat. Genet. 34, 187-192.

Ho, L., and Crabtree, G. R. (2010). Chromatin remodeling during development. Nature 463, 474-484.

Ho, L., Ronan, J. L., Wu, J., Staahl, B. T., Chen, L., Kuo, A., etal. (2009). An embryonic stem cell chromatin remodeling complex, esBAF, is essential for embryonic stem cell self-renewal and pluripotency. Proc. Natl. Acad. Sci. U.S.A. 106, 5181-5186.

Hohjoh, H., and Singer, M. F (1997). Sequence-specific singlestrand RNA binding protein encoded by the human LINE-1 retrotransposon. EMBO J. 16, 6034-6043.

$\mathrm{Hu}$, Y., Fisher, J. B., Koprowski, S., McAllister, D., Kim, M. S., and Lough, J. (2009). Homozygous disruption of the Tip60 gene causes early embryonic lethality. Dev. Dyn. 238 2912-2921.

Igarashi, S., Suzuki, H., Niinuma, T. Shimizu, H., Nojima, M., Iwaki, H., etal. (2010). A novel correlation between LINE-1 hypomethylation and the malignancy of gastrointestinal stromal tumors. Clin. Cancer Res. 16, 5114-5123.

Illingworth, R. S., and Bird, A. P. (2009). CpG islands - "a rough guide". FEBS Lett. 583, 1713-1720.

Jones, P. L., Veenstra, G. J., Wade, P. A. Vermaak, D., Kass, S. U., Landsberger, N., et al. (1998). Methylated DNA and $\mathrm{MeCP} 2$ recruit histone deacetylase to repress transcription. Nat Genet. 19, 187-191.

Kafri, T., Ariel, M., Brandeis, M., Shemer, R., Urven, L., McCarrey, J., et al. (1992). Developmental pattern of gene-specific DNA methylation in the mouse embryo and germ line. Genes Dev. 6, 705-714.

Kato, Y., Kaneda, M., Hata, K., Kumaki, K., Hisano, M., Kohara, Y., et al. (2007). Role of the Dnmt3 family in de novo methylation of imprinted and repetitive sequences during male germ cell development in the mouse. Hum. Mol. Genet. 16, 2272-2280.

Kato, S., Yokoyama, A., and Fujiki, R. (2011). Nuclear receptor coregulators merge transcriptional coregulation with epigenetic regulation. Trends Biochem. Sci. 36, 272-281.

Lander, E. S., Linton, L. M., Birren, B., Nusbaum, C., Zody, M. C., Baldwin, J., et al. (2001). Initial sequencing and analysis of the human genome. Nature 409, 860-921.

Laurent, L., Wong, E., Li, G., Huynh, T., Tsirigos, A., Ong, C. T., et al. (2010). Dynamic changes in the human methylome during differentiation. Genome Res. 20, 320-331.

Li, E., Bestor, T. H., and Jaenisch, R. (1992). Targeted mutation of the DNA methyltransferase gene results in embryonic lethality. Cell 69, 915-926.

Li, H., Rauch, T., Chen, Z. X., Szabó, P. E., Riggs, A. D., and Pfeifer, G. P. (2006). The histone methyltransferase SETDB1 and the DNA methyltransferase DNMT3A interact directly and localize to promoters silenced in cancer cells. J. Biol. Chem. 281, 19489-19500.

Liang, J., Wan, M., Zhang, Y., Gu, P., Xin, H., Jung, S. Y., et al. (2008). Nanog and Oct4 associate with unique transcriptional repression complexes in embryonic stem cells. Nat. Cell Biol. 10, 731-739.

Lister, R., Pelizzola, M., Dowen, R. H., Hawkins, R. D., Hon, G., TontiFilippini, J., et al. (2009). Human DNA methylomes at base resolution show widespread epigenomic differences. Nature 462, 315-322.

Loh, Y. H., Yang, L., Yang, J. C., Li, H., Collins, J. J., and Daley, G. Q. (2011). Genomic approaches to deconstruct pluripotency. Annu. Rev. Genomics Hum. Genet. 12, 165-185.

MacArthur, B. D., Ma'ayan, A., and Lemischka, I. R. (2009). Systems biology of stem cell fate and cellular reprogramming. Nat. Rev. Mol. Cell Biol. 10, 672-681.

Marfella, C. G., and Imbalzano, A. N. (2007). The Chd family of chromatin remodelers. Mutat. Res. 618, 30-40.

Martin, S. L., and Bushman, F. D. (2001). Nucleic acid chaperone activity of the ORF1 protein from the mouse LINE-1 retrotransposon. $\mathrm{Mol}$. Cell. Biol. 21, 467-475.

Meissner, A., Mikkelsen, T. S., Gu, H., Wernig, M., Hanna, J., Sivachenko, A., et al. (2008). Genome-scale DNA methylation maps of pluripotent and differentiated cells. Nature 454, 766-770.

Meshorer, E., Yellajoshula, D., George, E., Scambler, P. J., Brown, D. T., and Misteli, T. (2006). Hyperdynamic plasticity of chromatin proteins in pluripotent embryonic stem cells. Dev. Cell 10, 105-116. 
Mikkelsen, T. S., Hanna, J., Zhang, X., Ku, M., Wernig, M., Schorderet, P., etal. (2008). Dissecting direct reprogramming through integrative genomic analysis. Nature 454, 49-55.

Nagae, G., Isagawa, T., Shiraki, N., Fujita, T., Yamamoto, S., Tsutsumi, S., et al. (2011). Tissue-specific demethylation in CpG-poor promoters during cellular differentiation. Hum. Mol. Genet. 20, 2710-2721.

Nan, X., Ng, H. H., Johnson, C. A., Laherty, C. D., Turner, B. M., Eisenman, R. N., et al. (1998). Transcriptional repression by the methyl-CpGbinding protein $\mathrm{MeCP} 2$ involves a histone deacetylase complex. Nature 393, 386-389.

Okano, M., Bell, D. W., Haber, D. A., and Li, E. (1999). DNA methyltransferases Dnmt3a and Dnmt3b are essential for de novo methylation and mammalian development. Cell 99, 247-257.

Okano, M., Xie, S., and Li, E. (1998). Cloning and characterization of a family of novel mammalian DNA (cytosine-5) methyltransferases. Nat. Genet. 19, 219-220.

Phelan, M. L., Sif, S., Narlikar, G. J., and Kingston, R. E. (1999). Reconstruction of a core chromatin remodeling complex from SWI/SNF subunits. Mol. Cell 3, 247-253.

Rajasethupathy, P., Antonov, I., Sheridan, R., Frey, S., Sander, C., Tuschl, T., et al. (2012). A role for neuronal piRNAs in the epigenetic control of memory-related synaptic plasticity. Cell 149, 693-707.

Rauch, T. A., Zhong, X., Wu, X., Wang, M., Kernstine, K. H., Wang,
Z., et al. (2008). High-resolution mapping of DNA hypermethylation and hypomethylation in lung cancer. Proc. Natl. Acad. Sci. U. S. A. 105, 252-257.

Robertson, K. D. (2001). DNA methylation, methyltransferases, and cancer. Oncogene 20, 3139-3155.

Rubin, C. M., VandeVoort, C. A., Teplitz, R. L., and Schmid, C. W. (1994). Alu repeated DNAs are differentially methylated in primate germ cells. Nucleic Acids Res. 22, 5121-5127.

Schaniel, C., Ang, Y. S., Ratnakumar, K., Cormier, C., James, T., Bernstein E., et al. (2009). Smarcc1/Baf155 couples self-renewal gene repression with changes in chromatin structure in mouse embryonic stem cells. Stem Cells 27, 2979-2991.

Scott, A. F., Schmeckpeper, B. J., Abdelrazik, M., Comey, C. T., O’Hara, B. Rossiter, J. P., et al. (1987). Origin of the human L1 elements: proposed progenitor genes deduced from a consensus DNA sequence. Genomics 1, 113-125.

Shi, Y., Desponts, C., Do, J. T., Hahm, H. S., Schöler, H. R., and Ding, S. (2008). Induction of pluripotent stem cells from mouse embryonic fibroblasts by Oct4 and Klf4 with small-molecule compounds. Cell Stem Cell 3, 568-574.

Sims, R. J. III, Millhouse, S., Chen, C. F., Lewis, B. A., Erdjument-Bromage, H., Tempst, P., et al. (2007). Recognition of trimethylated histone $\mathrm{H} 3$ lysine 4 facilitates the recruitment of transcription postinitiation factors and pre-mRNA splicing. Mol. Cell 28, 665-676.
Su, J., Shao, X., Liu, H., Liu, S., Wu, Q., and Zhang, Y. (2012). Genome-wide dynamic changes of DNA methylation of repetitive elements in human embryonic stem cells and fetal fibroblasts. Genomics 99, 10-17.

Thomson, J. A., Itskovitz-Eldor, J. Shapiro, S. S., Waknitz, M. A., Swiergiel, J. J., Marshall, V. S., et al. (1998). Embryonic stem cell lines derived from human blastocysts. Science 282, 1145-1147.

Viré, E., Brenner, C., Deplus, R., Blanchon, L., Fraga, M., Didelot, C., et al. (2006). The Polycomb group protein EZH2 directly controls DNA methylation. Nature 439, 871-874.

Vissers, L. E., van Ravenswaaij, C. M. Admiraal, R., Hurst, J. A., de Vries, B. B., Janssen, I. M., et al. (2004). Mutations in a new member of the chromodomain gene family cause CHARGE syndrome. Nat. Genet. 36, 955-957.

Weber, M., Hellmann, I., Stadler, M. B., Ramos, L., Pääbo, S., Rebhan, M., et al. (2007). Distribution, silencing potential and evolutionary impact of promoter DNA methylation in the human genome. Nat. Genet. 39, 457-466.

Xie, H., Wang, M., BonaldoMde, F., Rajaram, V., Stellpflug, W., Smith, C., et al. (2010). Epigenomic analysis of Alu repeats in human ependymomas. Proc. Natl. Acad. Sci. U.S.A. 107, 6952-6957.

Xie, H., Wang, M., BonaldoMde, F., Smith, C., Rajaram, V., Goldman, S., et al. (2009). High-throughput sequence-based epigenomic analysis of Alu repeats in human cerebellum. Nucleic Acids Res. 37, 4331-4340.

Yamanaka, S. (2009). A fresh look at iPS cells. Cell 137, 13-17.

Zhu, D., Fang, J., Li, Y., and Zhang, J. (2009). Mbd3, a component of NuRD/Mi-2 complex, helps maintain pluripotency of mouse embryonic stem cells by repressing trophectoderm differentiation. PLoS ONE 4, e7684. doi: 10.1371/journal. pone. 0007684

Conflict of Interest Statement: The authors declare that the research was conducted in the absence of any commercial or financial relationships that could be construed as a potential conflict of interest.

Received: 05 June 2012; accepted: 17 October 2012; published online: 05 November 2012.

Citation: Hu Q and Rosenfeld MG (2012) Epigenetic regulation of human embryonic stem cells. Front. Gene. 3:238. doi: 10.3389/fgene. 2012.00238

This article was submitted to Frontiers in Epigenomics and Epigenetics, a specialty of Frontiers in Genetics.

Copyright (c) $2012 \mathrm{Hu}$ and Rosenfeld. This is an open-access article distributed under the terms of the Creative Commons Attribution License, which permits use, distribution and reproduction in other forums, provided the original authors and source are credited and subject to any copyright notices concerning any thirdparty graphics etc. 